\title{
15. INFLUENCE D'UN BOMBARDEMENT IONIQUE SUR DES JONCTIONS TUNNEL MÉTAL-SEMICONDUCTEUR
}

\section{G. SCHREDER et P. GUÉTIN}

Laboratoire d'Electronique et de Physique Appliquée, 94, Limeil, Brévannes, France

Nous avons soumis de l'arséniure de gallium de type $n$ à un bombardement d'ions de faible énergie avant l'évaporation du métal. Nous avons utilisé des gaz rares pour une gamme d'énergie de 60 à $210 \mathrm{eV}$.

Le fond continu en résistance croît avec l'énergie et ses variations avec la tension appliquée deviennent plus importantes. En considérant la dérivée seconde, il apparaît des structures dues à un effet tunnel inélastique et leurs réflexions par des anomalies du type
L. O. Une de celles-ci correspond au spectre des phonons acoustiques transverses de l'arséniure de gallium. Les autres structures dépendent du gaz utilisé et ne sont pas encore clairement définies. Pour des énergies plus faibles le bombardement ionique permet un nettoyage des surfaces et par la même occasion permet l'étude de la dépendance des anomalies avec l'état de surface du semiconducteur. 